\title{
Constitutional Dynamic Inhibition/Activation of Carbonic
}

\author{
Anhydrases \\ Dandan Su, ${ }^{\text {a, c }}$ Yan Zhang, ${ }^{\mathrm{b}}$ Sébastien Ulrich, ${ }^{\mathrm{c}}$ Mihail Barboiu, ${ }^{*, a}$ \\ ${ }^{a}$ Institut Europeen des Membranes, Adaptive Supramolecular Nanosystems Group \\ University of Montpellier, ENSCM-CNRS \\ Place E. Bataillon CC047, Montpellier, F-34095, France \\ E-mail: mihail-dumitru.barboiu@umontpellier.fr \\ ${ }^{\mathrm{b}}$ Key Laboratory of Carbohydrate Chemistry and Biotechnology, Ministry of \\ Education, School of Pharmaceutical Sciences \\ Jiangnan University \\ 1800 Lihu Avenue, Wuxi, 214122, P.R. China \\ ${ }^{\mathrm{c}}$ Institut des Biomolécules Max Mousseron (IBMM), CNRS, Université de \\ Montpellier, ENSCM, Montpellier, France.
}

\begin{abstract}
As one important member of metalloenzymes family, carbonic anhydrase (CA) is involved in the treatment of several diseases. Different approaches have therefore emerged to regulate the activity of CA, mostly acting on inner catalytic active site or outer microenvironment of the enzyme, leading to inhibition or activation of CA. In recent years, gradually increased attentions have been drawn into the adoption of constitutional dynamic chemistry (CDC) strategies for the screening and discovery of potent inhibitors or activators. The participation of reversible covalent bonds enabled the enzyme itself to select the optimal ligands obtained from diverse building blocks with comparatively higher degree of variety, resulting in the fittest recognition of enzyme ligands from complex dynamic systems. With the increasing implantation of CDC for enzyme target, it shows great potentials for the drug discovery- or environmental-oriented applications.
\end{abstract}

Keywords: Constitutional Dynamic Chemistry, Carbonic Anhydrase, Constitutional Frameworks 


\section{Introduction}

Constitutional Dynamic Chemistry (CDC) and its application Dynamic Combinational Chemistry $(\mathrm{DCC})^{[1]}$ provide diverse opportunities for screening the chemical space at molecular and supramolecular levels for dedicated applications in chemistry, medicine and materials sciences. By using dynamic constitutional systems constructed by combination of reversible interacting components, it is possible to use various stimuli, such as light, heat, $\mathrm{pH}$ or ionic gradients, leading, in the presence of templating or molding agents, to self-selection or optimization of the fittest constituents.

In the last decades, $\mathrm{DCC}^{[1]}$ dealing with the studies of dynamic chemical libraries built on reversible covalent interactions, showed bright prospects in diverse applications such as drug discovery, ${ }^{[2,3]}$ materials science, ${ }^{[4,5]}$ gene delivery, ${ }^{[6,7]}$ catalyst screening, ${ }^{[8,9]}$ etc. Among these, enzyme catalysis has attracted increasing attention from incipient development of the field. For example, DCC has been applied for highly selective enzyme-catalyzed asymmetric synthesis, resulting in identification of the optimal substrates fitting to the target enzyme active sites. ${ }^{[10-13]}$ Moreover, the catalytic activity of enzymes can be tuned effectively with the participation of dynamic constitutional frameworks, ${ }^{[14]}$ as well as discrete enzyme classification through pattern recognition methodology. ${ }^{[15]}$

As a type of most commonly used metalloenzymes, carbonic anhydrase (CA, EC 4.2.1.1) has been selected and integrated with dynamic constitutional systems for the screening and identification of its inhibitors and activators. CAs are zinc-containing metalloenzymes, which are encoded by five gene families, including $\alpha$-CAs, $\beta$-CAs, $\gamma$-CAs, $\delta$-CAs and $\zeta-\mathrm{CAs}$, with diverse catalytic activity and tissue distribution. ${ }^{[16]}$ This superfamily plays a vital role in maintaining a stable body environment by catalyzing the carbon dioxide hydration reaction. ${ }^{[17,18]}$ Therefore, the tuning of catalytic activity of CAs became a key breakthrough point for the treatment of several diseases. For example, the inhibitors of CAs can reduce the production of bicarbonate and lower the intraocular pressure, which is mainly used in the clinical treatment of glaucoma. ${ }^{[19-22]}$ In addition, the treatment of tumors, epilepsy, acute mountain 
sickness and obesity are inseparable from the effects of carbonic anhydrase inhibitors (CAIs). ${ }^{[23-27]}$ As for carbonic anhydrase activators (CAAs), they may improve memory and learning ability, paving the way for the treatment of Alzheimer's disease. ${ }^{[28,29]}$

More specifically, the design of CAIs and CAAs usually involve the inner active site or the outer surrounding microenvironment of CA. It has emerged that CAIs can be mostly developed through direct coordination binding with the metal ion - zinc, which is playing the key role for catalysis inside the active site of the CA. ${ }^{[18]}$ Meanwhile, the development of CAAs has been, comparatively, much slower, probably due to their complicated pharmacology and catalytic cycle. It has been suggested that the location of CAAs in the entrance of enzyme active site cavity is essential for activating enzyme activity, leading to acceleration of the proton-transfer step via intermediate water-wires. ${ }^{[18,30]}$ Additionally, the external microenvironment of enzyme can also make a difference for enhancing the activity of CAs and need to be further studied for developing continuous processes for medical or environmental applications. ${ }^{[31]}$ In both cases, DCC can provide efficient approaches for the screening of optimal molecular or supramolecular components from various dynamic constitutional systems, in order to inhibit or activate the catalytic activity of CAs.

Herein, we have summarized a set of CA inhibitors and activators which were identified through the DCC strategy. The aim is to highlight recent advances of dynamic constitutional systems with carbonic anhydrases as the target enzyme. Representative examples will be illustrated below, hoping to inspire new ideas for the application of drug discovery or enzyme immobilization for environmental applications.

\section{Enzyme inhibition}

The active site of $\mathrm{CA}$ is mainly constructed around the $\mathrm{Zn}^{2+}$ cation, which is located at the bottom of the active pocket and coordinated by His94, His96 and His119 and a water molecule. ${ }^{[18,24]}$ During the general catalytic cycle (Fig. 1), the water molecule is first dissociated into proton and hydroxyl ions under neutral $\mathrm{pH}$ 
conditions prior to nucleophilic addition of the latter onto carbonic acid, generating bicarbonate, and proton-transfer of the former to regenerate the zinc-water coordination complex. This proton-transfer step is rate-limiting and therefore represent a leverage tool for designing CAI and CAA. ${ }^{[32,33]}$ Regarding the design of CA inhibitors, not only the zinc-binding groups, but also other enzyme attaching moieties, such as the $\mathrm{Zn}^{2+}$ coordinated water and the entrance to the active site, can be used as the action site. ${ }^{[34]}$ Until now, five inhibition mechanisms of CA has been reported, ${ }^{34}$ leading to demonstrated roles of CAI in numerous diseases, including glaucoma, epilepsy, obesity and kidney cancer. ${ }^{[35-39]}$
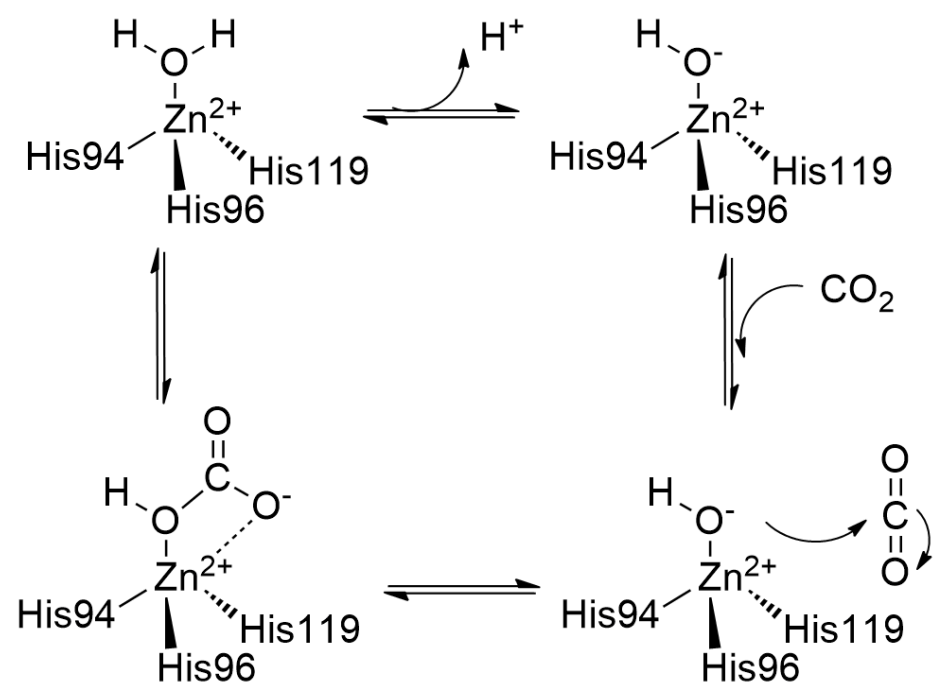

Fig. 1 The catalytic mechanism of the carbon dioxide hydration reaction by CAs. ${ }^{[18]}$

Lehn and Huc were the first group reporting a set of hydrophobic sulfonamide-based CAIs generated by reversible imine combinations of constitutional dynamic libraries composed of amine and aldehyde components. They have been demonstrated that a known aromatic sulfonamide CAI, is amplified from the combinatorial mixture of components, based on its favorable affinity toward bCAII, ${ }^{[40,}$ ${ }^{41]}$ via the tetrahedral coordination with the sulfonamide group to the $\mathrm{Zn}^{2+}$ center. ${ }^{[18]}$ Thereafter, other classes of sulfonamides CAIs and derivatives were exploited for the CA recognition efficiency through the binding with the enzyme active site. ${ }^{[2,43]}$ Meanwhile, their X-ray crystallography were also disclosed since the first sulfonamides CAI was found through X-ray crystallography of the obtained 
enzyme-inhibitor adduct. ${ }^{[44]}$ In this context, a series of CAIs have been obtained and extended from the DCC approach to target different CA isoforms. They have been illustrated below (Table 1), classified by different reversible bonds, including aminocarbonyl/imine interconversion, alkene cross metathesis and acyl hydrazones.

Table 1. Representative reversible bonds for the dynamic generation of CAIs targeting carbonic anhydrases

\begin{tabular}{lccc}
\hline Regulation & Reversible bonds & Targeted CA & Ref \\
\hline Inhibition & Alkene cross metathesis & bCAII & {$[45]$} \\
& $\begin{array}{c}\text { Acyl hydrazones } \\
\text { Amino-carbonyl/imine } \\
\text { interconversion }\end{array}$ & bCAII & {$[46]$} \\
& hCAI, hCAII, hCAIX & ${ }^{[47-50]}$ \\
\hline
\end{tabular}

Amino-carbonyl/imine interconversion under thermodynamic control is one of the most popular dynamic covalent chemistry used for these purposes. Our group have used various aldehydes and amines to form stable Schiff base in a constitutional dynamic library (Fig. 2), for the purpose of corelating the inhibitory power of CAIs with their respective amplification is solution, useful for understanding the role of multivalent interactions with the enzyme toward the discovery of novel DCC CAIs. ${ }^{[47,49]}$ Several active compounds from a pool of twenty components can be efficiently identified with the amplification of the most active CAIs, 1C, 1D, 2D (Fig. 2) together with less active CAIs $\mathbf{1 E}$ or $\mathbf{4 E}$ which are probably forming via the outer side of the enzyme surface-directed reactions or via kinetic-rapid reactions in solution. We have next showed that the flexible amines obtained by $\mathrm{NaBH}_{3} \mathrm{CN}$ reduction of imine adducts showed better performance on hCAI inhibition, while the rigid imine expressed higher selectivity towards hCAII over hCAI (Fig. 3). ${ }^{[48]}$

At this point, it is important to highlight those reactions occurring in the active site are of outmost importance, but they may be related to the favourable diffusion and the oriented binding of the components within the active site. Parallel side reactions cand be favoured by respective binding of components on other regions situated out of active enzymatic centre. They are providing "parasite" reactions to be 
considered useful for the selection features of the combinatorial systems under considerations.

Supuran group has designed a series of sulfanilamide derivatives, while using imine Schiff bases chemistry to probe the structure-activity relationship of CA inhibitors, thus providing efficient screening of the most effective inhibitors for CA isoforms. ${ }^{[50-53]}$ The precise position of the interactional groups on the molecular platform is highly important, substantially affecting the functional binding of the CAI within the active center. The impact of sulfonamide group position on the phenyl ring has been studied through a stopped-flow $\mathrm{CO}_{2}$ hydrase assay, indicating that smaller $K_{\mathrm{I}}$ values for hCAI and hCAII can be obtained from para position than meta- and orthopositions, under the condition of the same substituents on the isatin ring. They also illustrated the docking position in the active site of hCAIX with hydrogen bonds formed between Gln 92 and the imine group. In the end, compound a meta-substituted aromatic sulfonamide group presented the highest selectivity towards the tumor-associated isozyme hCAIX, compared to the cytosolic hCAI and hCAII. ${ }^{[50]}$

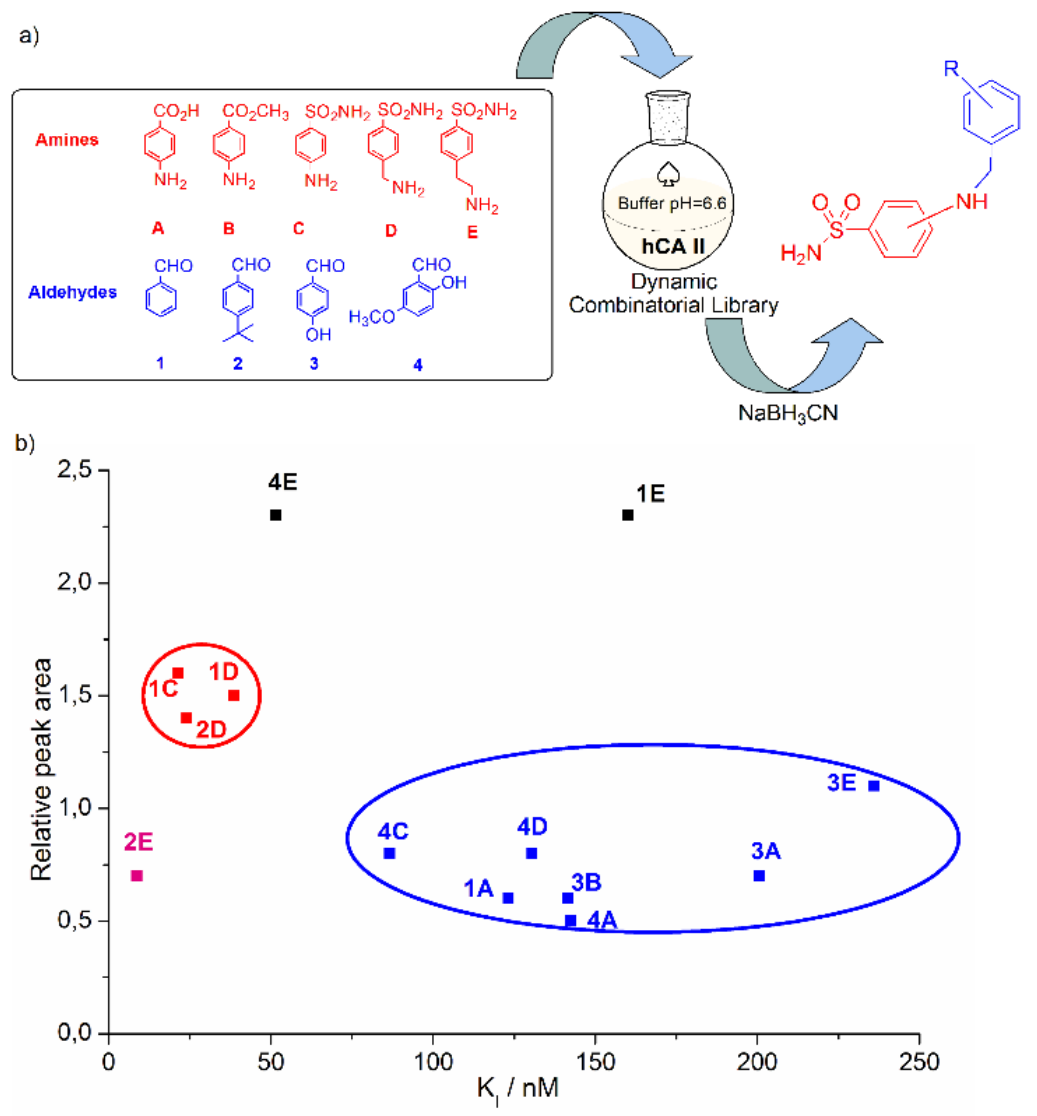


Fig. 2 a) Constitutional dynamic library of amino, A-E and aldehyde, 1-4 components towards the discovery of isozyme-specific inhibitors; (b) Relative peak area expressing the amplification of the DCL function of inhibitory power (KI) against hCA II. ${ }^{[47]}$

Considering the pharmacological importance for the discovery of isoform-selective CAIs, hCAI and hCA II, have been subjected to a parallel screening of the same CDL. The use of parallel constitutional screening of CDL chemistry for the discovery of enzyme inhibitors is straightforward method that provides insights toward the generation of efficient classes of selective, high affinity inhibitors. These results by our group ${ }^{[49]}$ also point to the possibility of modulating the drug discovery methods by constitutional recomposition induced by a specific enzymatic target (Fig. 3). The structurally fittest CAIs have been found for each isozyme, illustrating that the response a CDC system may evolve differently to the presence of two different enzymatic effects. This example is demonstrating variable behaviours of CDC systems, showing precise adaptation under the pressure of variable external factors.



Fig. 3 Inhibition constants $K_{I}$ and the amplification of the constitutional dynamic library (CDL) against catalytically active human hCA I and hCA II cytosolic isosymes. ${ }^{[49]}$

Aiming for the dual functionality, aromatic sulphonamideacyl-hydrazone can be combined with various aldehydes and used for the generation of a dynamic combinatorial library towards selection of bCAII inhibitors (Fig. 4a). ${ }^{[46]}$ Amino acid derivatives were included into the dynamic system through hydrazone exchange 
reactions, to provide extra periphery recognition with the targeting enzyme. Then the screening of enzyme-ligand complexes was performed directly from the dynamic combinatorial library with Fourier transform ion cyclotron resonance mass spectrometry. As a result, the sulfonamide-amino acid conjugates with acyl-hydrazone linkages presented developable potential as CA inhibitors. Furthermore, Poulsen group reported a pre-equilibrated dynamic combinatorial library (pDCL) based on reversible alkene metathesis (Fig. 4b). ${ }^{[45]}$ In this design, allyl ester benzene sulfonamide, which composed with an allyl substituent for terminal alkene metathesis and an aromatic sulfonamide moiety for bCAII recognition, was used to construct the dynamic scaffolds through the reversible reaction with ten additional building blocks respectively. Regarding to the fragment-based drug discovery to bCAII inhibitors, it was demonstrated that the cross-metathesis compound with a terminal acetate group, as well as homodimer and trans-isomers, exhibited more potent bCAII binding affinity with a lower equilibrium dissociation constant. Meanwhile, heterogeneous catalysis showed advantages on the convenience and benefits over homogeneous catalysis.

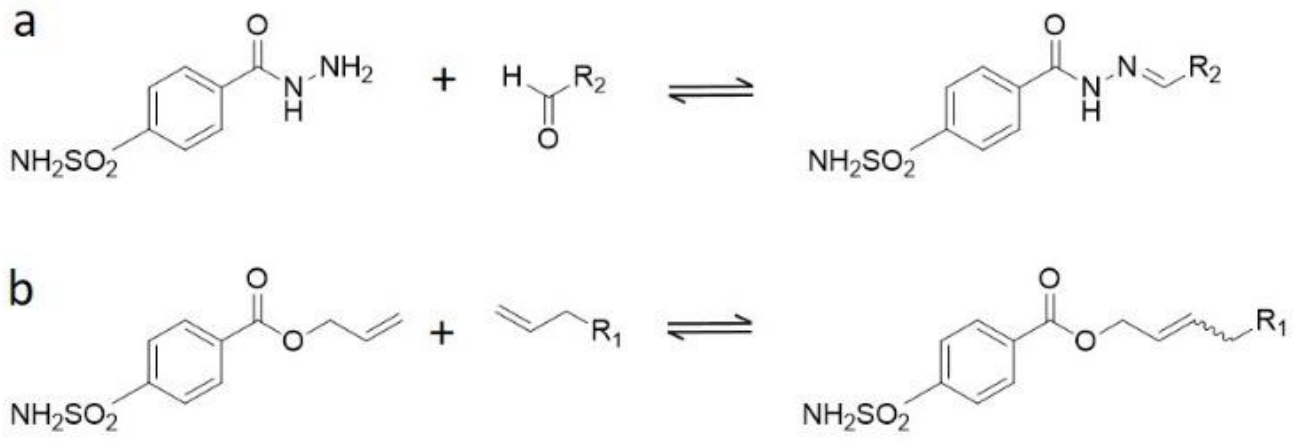

Fig. 4 Generation of the DCL with (a) acyl hydrazones exchange ${ }^{[46]}$ and (b) alkene cross-metathesis reaction. ${ }^{[45]}$

\section{Enzyme Activation}

It has been reported that CA level may have influences on the memory therapy as well as spatial learning, thus, the tuning of CA activity can be important for the related treatments. ${ }^{[29,54]}$ However, no CAAs have been exploited for clinical applications because of their complicated pharmacology and catalytic cycle. Considering the 
design of CAAs, it has been suggested that those CAAs ligands are usually located at the entrance of enzyme active site cavity. ${ }^{[30]}$ For instance, spectroscopic evidence provided by X-ray crystallography showed that histamine, which is indeed located at the entrance of enzyme active site, exhibited activation of human CA isoform (hCAII) through the imidazole moiety which accelerated the proton-transfer rate-determining step of the CA catalytic cycle. ${ }^{[30,55]}$ Furthermore, selective CAAs have been demonstrated with the structure of ureido/bis-ureido histamine derivatives, which showed activation effect towards hCAI. ${ }^{[56]}$

Since the screening efficiency is relatively low by designing and synthesizing diverse ligands one by one, dynamic combinatorial approach was adopted to accelerate the process. Lehn and Ramström have reported an efficient screening method with Dynamic Deconvolution, ${ }^{[57,58]}$ to select and amplify the active molecules for enzyme activations. During the procedure, a parallel screening among diverse building blocks was carried out, by removing the components one by one from the final dynamic combinatorial library and test the activity of the enzyme. If the removed component is active an important effect in the catalytic enzyme activity is observed. With all these in mind, we combined agonistic inhibitor and activator components within different DCLs mixtures. Dynamic dominant behaviours in the expression of human of Carbonic Anhydrase hCA I activity are observed, most probably related to the differences in the long-distance binding behaviors of the of CAAs compared to the stronger direct binding of CAIs to $\mathrm{Zn}^{2+}$ in the active enzyme pocket. The relative activity of the mixtures reveals the inhibitory effects dominate over the activating ones in DCLs containing both inhibitors and activators of the hCA I. ${ }^{[59]}$

Apart from the active site, the external enzyme microenvironment is also worth to be exploited, since it is essential for the catalytic activity and selectivity of the enzymes. ${ }^{[60,61]}$ In this respect, dynamic constitutional chemistry has also found its applications in screening optimal three-dimensional frameworks reversibly connecting at molecular and supramolecular levels for better enzyme activation effect.

Dynamic reversible connections between components can be transferred from A-B binary type of combinations as previously described before toward 2D-3D 
Dynamic Constitutional Frameworks DCFs, constructed from bi(tri)functional components and core connectors via different covalent reversible chemistries. ${ }^{[60]}$

For example, the direct addition of PEG-ylated dynamic constitutional frameworks linked with reversible imine bonds at equipped with $\mathrm{H}$-bonding motifs, showed enzyme activation effect towards CA. ${ }^{[31,62,63]}$ The mechanism can be that the in situ generation of dynamic framework via imine, bond formation simultaneously encapsulate CA through hydrogen-bonding and supplementary imino-bond formation with the amino groups on the enzyme surface. They are generating an adaptive protective layer around the enzyme, leading to more stabilized tertiary protein structures and they are providing proximal multivalent proton acceptor groups in the close proximity of the enzyme surface, assisting the proton shuttling during the catalytic cycle (Fig. 5).

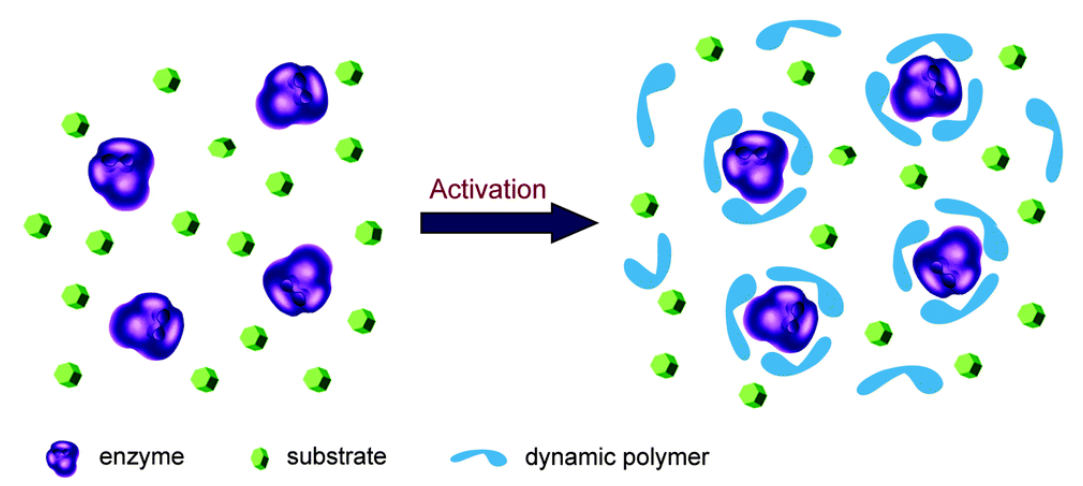

Fig. 5 Concept of enzyme activation via dynamer encapsulation. ${ }^{[31]}$

Our group has pioneered this field by reporting a straight-forward constitutional enzyme activation strategy, using dynamic constitutional frameworks as enzyme microenvironment activators (Fig. 6). ${ }^{[31]}$
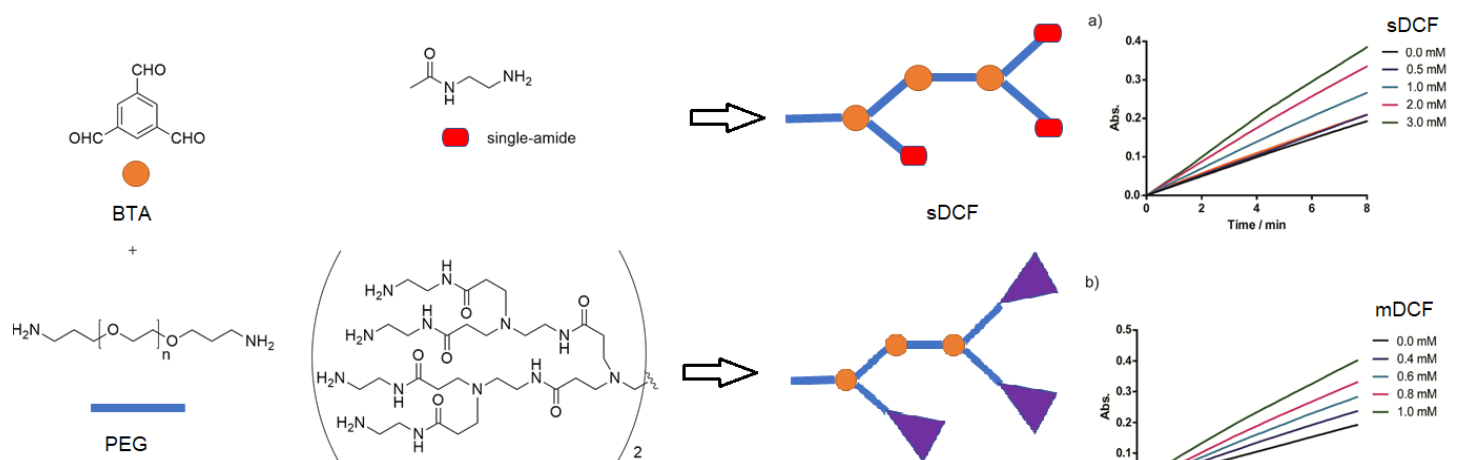

PEG
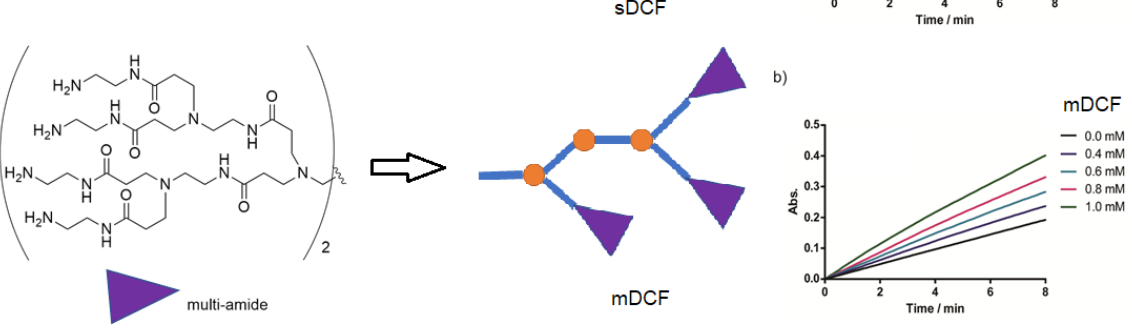
Fig. 6 Dynamic Enzyme Activation in Multivalent H-bonding DCFs composed of core centers, PEG connectors and (a) single-amide head, sDCF and b) multi-amide head mDCF. Enzymatic activity of bCA on hydrolysis reaction of $p$-nitrophenyl acetate, was determined by using UV absorbance at $400 \mathrm{~nm}$ recorded within time for different concentrations of DCFs relative to the concentration of the BTA core center [31]

The DCFs were composed from benzenetrialdehyde, BTA core centers, water soluble polyethyleneglycol $\left(\mathrm{PEG}_{1500}\right)$ connectors and mono or dendrimeric amide-head components. The only former H-bonding components show a very low activity, while they became very active when they are multivalently assembled within DCFs. Interestingly the former PEGylated network, despite its strong interactions binding probably via imine-bond formation on the enzyme surface, did not enhance the CA activity. ${ }^{[31]}$ Further results showed that the multi-amide substituted dendrimer (PAMAM) provided higher activation effect towards CA than single-amide substituted building blocks, emphasizing the importance of multivalent presentations of the active sites as proton sponges within DCFs. The enzyme catalytic activities were determined to be $1.7 \mathrm{mM}$ sDCF and $0.62 \mathrm{mM}$ MDCF respectively, presenting moderate activation effect compared to molecular activators, but attributed to the number of H-bonding interactions with enzyme surface and the facilitated proton shuttling.

Next, we changed the target enzyme from carbonic anhydrase to lipase, the catalytic enhancement effect on enzyme catalytic activity is still observed suggesting that observed effect is broad and effective potential applications with the DCFs-enzyme systems can be developed in the future. The shell-like encapsulation of the enzyme through the multivalent interactions between DCF and enzymes is most probably connected to the presence of the PEGylated matrix, contributing to the whole lipase interfacial activity enhancement. With such combined effects, the amide-PEG-DCFs accelerate lipase-catalyzed hydrolysis with high efficiencies. ${ }^{[64]}$ 

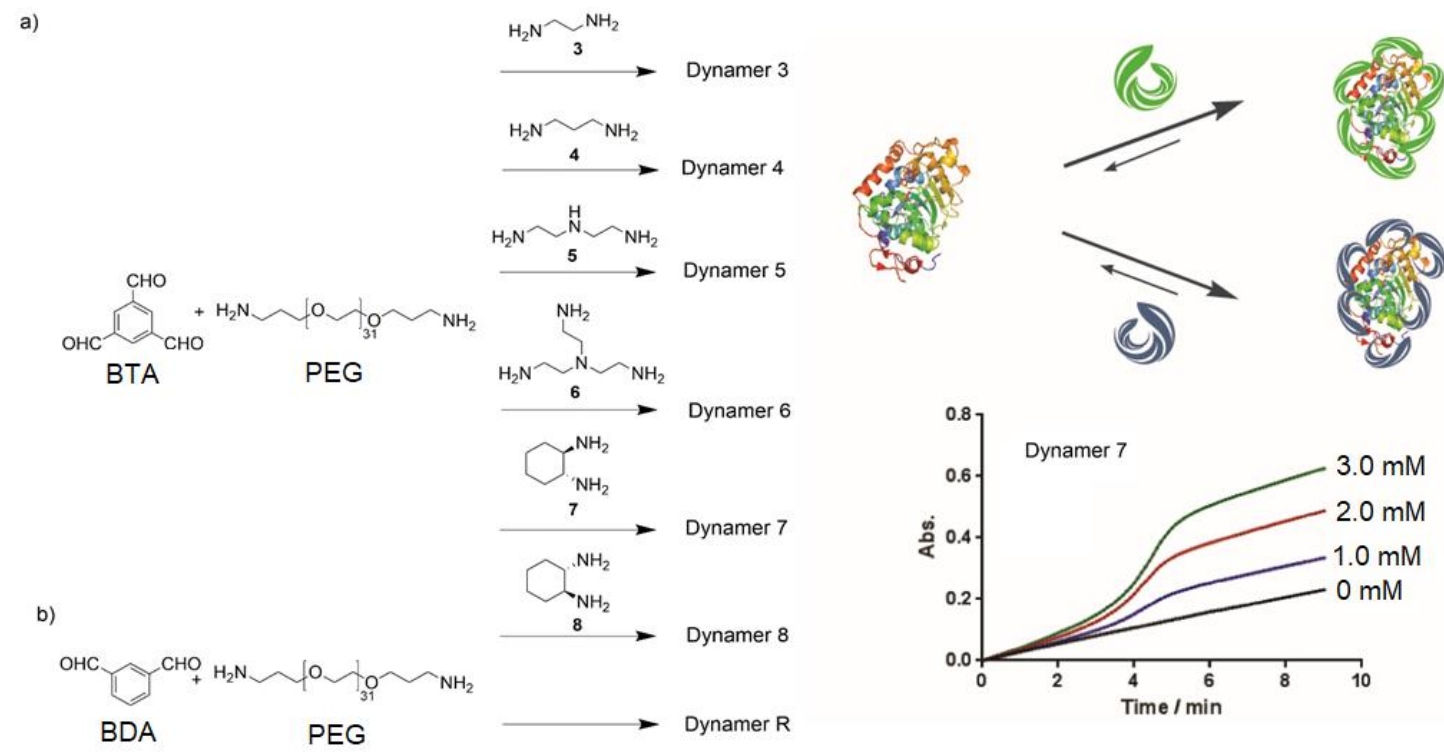

Fig. 7. Exponential activation of Carbonic Anhydrase by encapsulation in a) DCFs host-matrices with stereochemical and chiral discrimination. Activity change of bCA on the hydrolysis reaction of p-nitrophenyl acetate, with the addition of a dynamer 7 (concentrations relative to 1 ) followed by UV-vis spectroscopy. ${ }^{[62]}$

Linear, cross-linked and optically active DCFs have been synthetized by including of diamino components, leading to an exponential activation of the bCA enzyme catalytic activity. While the DCFs-enzyme interaction is considered as shell-like matrix around the enzyme, the asymmetric DCFs may generate a chiral micro-environment for the enzyme encapsulation, leading to high exponential enhancement of the enzyme activity. ${ }^{[62]}$

The nature and structural behaviors of functional groups used for the DCFs synthesis are highly important and their influence on CA activation has been further explored by using an efficient enzyme self-screening system, in order to obtain optimized microenvironment of CA (Fig. 8). In a given dynamic system, the interactions between CA and the functional DCFs were controlled by the competition between protein-DCF affinity and the direct DCFs formation via imine cross-linking reactions. Based imine bond formation with the amine groups and via non-covalent interactions on the enzyme iso-surface a re-equilibration can be achieved upon the addition of CA. It was found that lower incorporation of small amine components in DCFs exhibited higher activation effects, owing to the enzyme selection preferences. 
The enzyme structure directed DCF formation kinetic studies were in good correlation with their activation effects, providing insights into the CA-substrate interactions upon addition of the DCFs. ${ }^{[63]}$ This constitutional dynamic strategy offers a new opportunities for enzyme self-directed microenvironment optimizations and further tuning of enzyme activities.



Fig. 8 General concept of enzyme-driven microenvironment optimization by dynamic constitutional imine systems. ${ }^{63}$

\section{Summary and outlook}

Over the past few decades, dynamic constitutional strategies have been emerged to generate enzyme-dynamic combinatorial systems that have been used in drug discovery- or environmental-oriented applications. The convergent combinatorial synthetic strategy uses simple synthetic building blocks with participation of reversible molecular and supramolecular linkages to provides a parallel and facile screening of complex mixtures of appropriate ligands or external networks from the adaptive constitutional polymers. It allows variability, versatility and easy screening for the best performances.

With Carbonic Anhydrase-CA as the target enzyme, both inhibitors and activators have been widely explored and the using dynamic constitutional chemistry may led to efficient easy discovery and rapid identification of highly efficient CAIs and CAAs candidates. It is worth noting that not only enzyme active site but also the surrounding microenvironment is essential for the regulation of enzyme activity.

The examples discussed here illustrated the developing advances with regarding to inhibitors and activators of CA, through the generation of dynamic adaptive 
libraries. Different enzyme-ligands complexes have been classified by the ligand architectures, as well as the type of reversible linkages. The inhibition capacity of small dynamic molecules towards CA were realized through binding at the inner enzyme active site. On the other hand, as for the design of activators of CA, dynamic constitutional frameworks composed of diverse building blocks were self-screened for the optimal outer microenvironment with the presence of CA, with activation effects further verified. It leaves the enzyme the freedom to select its fittest effector.

As an important disease-related enzyme target, CA has long attracted a lot attention from researchers, and the search of its inhibitors and activators has been continuously conducted. In this process, dynamic constitutional chemistry has showed high efficiency and demonstrated broader application potentials. However, there is still some space for further exploration. For example, ligands for several CA isoforms, including hCAI, hCAII, hCAIX and bCAII, have been screened, while others still remain to be studied. Operate the inner active pocket or outer enzyme/protein environment should constitute an important endeavor for future research activities in this field. Moreover, the exact activation and inhibition mechanism still need be explained through conformations of enzyme-dynamer structures. Therefore, further innovations can bring more exciting improvements to this field.

Conflicts of interest "There are no conflicts to declare".

Acknowledgements : DS appreciates receiving a scholarship from China Scholarship Council as support at University of Montpellier, France. This work was supported by M-ERA NET 2019 grant to MB via Agence Nationale de la Recherche ANR-20-MERA-0001-01, SMARTMATTER. YZ thanks financial support from Natural Science Foundation of Jiangsu Province (BK20180625) and National First-class Discipline Program of Light Industry Technology and Engineering (LITE2018-20).

\section{References}

1 Constitutional Dynamic Chemistry, Topics in Current Chemistry, M. Barboiu Editor, Springer Verlag, 2012. ISBN 978-3-642-28344-4

2 O. Ramström and J.-M. Lehn, Nat. Rev. Drug Discov., 2002, 1, 26-36. 
3 Y. Zhou, C. Li, J. Peng, L. Xie, L. Meng, Q. Li, J. Zhang, X. D. Li, X. Li, X. Huang, X. Li, J. Am. Chem. Soc., 2018, 140, 15859-15867.

4 Z. Zhang, X. Wang, Y. Wang and J. Hao, Biomacromolecules, 2018, 19, 980-988.

5 L. Yue, S. Wang, V. Wulf, I., Nat. Commun., 2019, 10, 4774.

6 C. Bouillon, Y. Bessin, F. Poncet, M. Gary-Bobo, P. Dumy, M. Barboiu, N. Bettache and S. Ulrich, J. Mat. Chem. B, 2018, 6, 7239-7246.

7 L. Marin, D. Ailincai, M. Calin, D. Stan, C. A. Constantinescu, L. Ursu, F. Doroftei, M. Pinteala, B. C. Simionescu and M. Barboiu, ACS Biomater. Sci. Eng., 2016, 2, 104-111.

8 F. Schaufelberger and O. Ramström, J. Am. Chem. Soc., 2016, 138, 7836-7839.

9 F. Schaufelberger, B. J. J. Timmer and O. Ramström, Chem. Eur. J., 2018, 24, 101-104.

10 Y. Zhang, Y. Zhang and O. Ramström, , Catal. Rev., 2020, 62, 66-95.

11 Y. Zhang, P. Vongvilai, M. Sakulsombat, A. Fischer and O. Ramström, Adv. Synth. Cat., 2014, 356, 987-992.

12 Y. Zhang, L. Hu and O. Ramström, Chem. Commun., 2013, 49, 1805-1807.

13 L. Hu, F. Schaufelberger, Y. Zhang and O. Ramström, Chem. Commun., 2013, 49, 10376-10378.

14 Y. Zhang, W.-X. Feng, Y.-M. Legrand, C. T. Supuran, C.-Y. Su and M. Barboiu, Chem. Commun., 2016, 52, 13768-13770.

15 Y. Zhang, H. S. N. Jayawardena, M. Yan and O. Ramström, Chem. Commun., 2016, 52, 5053-5056.

16 C. T. Supuran, Carbonic anhydrase inhibitors, Bioorg. Med. Chem. Lett., 2010, 20, 3467-3474..

17 A. Scozzafava and C. T. Supuran, J. Enz. Inh. Med. Chem., 1999, 14, 343-363.

18 C. T. Supuran, Nat. Rev. Drug Discov., 2008, 7, 168-181

19 C. T. Supuran, A. S. A. Altamimi and F. Carta, Exp. Op. Therap. Pat., 2019, 29, 781-792.

20 M. Fares, W. M. Eldehna, S. Bua, C. Lanzi, L. Lucarini, E. Masini, T. S. Peat, H. A. Abdel-Aziz, A. Nocentini, P. A. Keller and C. T. Supuran, J. Med. Chem., 2020, 63, 3317-3326.

21 S. Kalinin, A. Valtari, M. Ruponen, E. Toropainen, A. Kovalenko, A. Nocentini, M. Gureev, D. Darin, A. Urtti, C. T. Supuran and M. Krasavin, Bioorg. Med. Chem., 2019, 27, 115086.

22 M. Barboiu, C.T. Supuran, L. Menabuoni, A. Scozzafava, F. Mincione, F. Briganti, G. Mincione, J. Enzyme. Inhib. 1999, 15, 23-46.

23 A. Scozzafava, C. T. Supuran and F. Carta, Exp. Op. Therap. Pat., 2013, 23, $725-735$.

24 C. T. Supuran, J. Enz. Inh. Med. Chem., 2018, 33, 485-495.

25 C. T. Supuran, Exp. Op. Therap. Pat., 2018, 28, 709-712.

26 A. Nocentini and C. T. Supuran, Exp. Op. Therap. Pat., 2018, 28, 729-740.

27 C. T. Supuran, Exp. Op. Therap. Pat., 2016, 12, 423-431.

28 W. A. Meier-Rüge, P. Iwangoff and K. D. Reichlmeier, Arch. Geron. Geriatr., 1984, 3, 161-165. 
29 M.-K. Sun and D. L. Alkon, Trends Pharmacol. Sci., 2002, 23, 83-89.

30 C. T. Supuran, Future Med Chem., 2018, 10, 561-573.

31 Y. Zhang, Y.-M. Legrand, E. Petit, C. T. Supuran and M. Barboiu, Chem. Commun. 2016, 52, 4053-4055.

32 C. Luca, M. Barboiu, C.T. Supuran, Rev. Roum. Chimie, 1991, 36, 1169-1173.

M. Barboiu, C.T. Supuran, L. Menabuoni, A. Scozzafava, F. Mincione, F. Briganti, G. Mincione, J. Enzyme. Inhib. 1999, 15, 23-46.

33 J. F. Domsic, B. S. Avvaru, C. U. Kim, S. M. Gruner, M. Agbandje-McKenna, D. N. Silverman and R. McKenna, J. Biol. Chem., 2008, 283, 30766-30771.

34 C. T. Supuran, Exp. Op. Therap. Pat., 2017, 12, 61-88.

35 M. Barboiu, C. T. Supuran, L. Menabuoni, A. Scozzafava, F. Mincione, F. Briganti and G. Mincione, J. Enz. Inh. Med. Chem., 1999, 15, $23-46$.

36 N. Krall, F. Pretto, W. Decurtins, G. J. L. Bernardes, C. T. Supuran and D. Neri, Angew. Chem. Int. Ed., 2014, 53, 4231-4235.

37 C. T. Supuran, Exp. Op. Drug Met. Tox., 2020, 16, 297-307.

38 C. T. Supuran, Exp. Op. Emerg.Drugs, 2012, 17, 11-15.

39 C. Temperini, A. Innocenti, A. Scozzafava, S. Parkkila and C. T. Supuran, J. Med. Chem., 2010, 53, 850-854.

40 I. Huc and J.-M. Lehn, V Proc. Natl. Acad. Sci USA, 1997, 94, 2106-2110.

41 R. Nguyen and I. Huc, Angew. Chem. Int. Ed., 2001, 40, 1774-1776.

42 S. Pastorekova, S. Parkkila, J. Pastorek and C. T. Supuran, J. Enz. Inh. Med. Chem., 2004, 19, 199-229.

43 C. T. Supuran and A. Scozzafava, Curr. Med. Chem. - Immunol., Endocrine \& Metabol. Ag., 2001, 1, 61-97.

44 C. T. Supuran, J. Enz. Inh. Med. Chem., 2012, 27, 759-772.

45 S.-A. Poulsen and L. F. Bornaghi, Bioorg. Med. Chem. 2006, 14, 3275-3284.

46 S.-A. Poulsen, J. Am. Soc. Mass Spectrom., 2006, 17, 1074-1080.

47 G. Nasr, E. Petit, C. T. Supuran, J.-Y. Winum and M. Barboiu, Bioorg. Med. Chem. Lett., 2009, 19, 6014-6017.

48 G. Nasr, A. Cristian, M. Barboiu, D. Vullo, J.-Y. Winum and C. T. Supuran, Bioorg. Med. Chem. 2014, 22, 2867-2874.

49 G. Nasr, E. Petit, D. Vullo, J.-Y. Winum, C. T. Supuran and M. Barboiu, J. Med. Chem., 2009, 52, 4853-4859.

50 Ö. Güzel-Akdemir, A. Akdemir, N. Karalı and C. T. Supuran, Org. Biomol. Chem., 2015, 13, 6493-6499.

51 N. Singasane, P. S. Kharkar, M. Ceruso, C. T. Supuran and M. P. Toraskar, IJ. Enz. Inh. Med. Chem., 2015, 30, 901-907.

52 A. S. El-Azab, A. A.-M. Abdel-Aziz, S. Bua, A. Nocentini, M. M. Alanazi, N. A. AlSaif, I. A. Al-Suwaidan, M. M. Hefnawy and C. T. Supuran, Bioorg. Chem., 2019, 92, 103225 .

53 M. Durgun, H. Turkmen, M. Ceruso and C. T. Supuran, Bioorg. Medicinal Chem. Lett., 2015, 25, 2377-2381.

54 W. Meier-Ruge, P. Iwangoff and K. Reichlmeier, Archiv. Gerontol. Geriatrics, 1984, 161-165. 
55 F. Briganti, S. Mangani, P. Orioli, A. Scozzafava, G. Vernaglione and C. T. Supuran, Biochemistry, 1997, 36, 10384-10392.

56 E. Licsandru, M. Tanc, I. Kocsis, M. Barboiu and C. T. Supuran, A J. Enz. Inh. Med. Chem., 2017, 32, 37-46.

57 T. Bunyapaiboonsri, O. Ramström, J.-M. Lehn, L. Peng and Lohmann,Sophie and Goeldner, Maurice, Chembiochem, 2001, 2, 438-444.

58 O. Ramström, S. Lohmann, T. Bunyapaiboonsri and J.-M. Lehn, Chem. Eur. J., 2004, 10, 1711-1715.

59 M. Y. M. Abdelrahim, M. Tanc, J.-Y. Winum, C. T. Supuran and M. Barboiu, Chem. Commun., 2014, 50, 8043-8046.

60 Y. Zhang, Y.-C. Qi, S. Ulrich, M. Barboiu and O. Ramström, Mater. Chem. Front., 2020, 4, 489-506. DOI: 10.1039/C9QM00598F.

61 M. Bilal, J. Cui and H. M. N. Iqbal, Int. J. Biol. Macromol., 2019, 130, 186-196.

62 Y. Zhang, C. T. Supuran and M. Barboiu, Chem. Eur. J., 2018, 24, 715-720.

63 Y. Zhang, M. Barboiu, O. Ramström and J. Chen, ACS Catalysis, 2019, 10, $1423-1427$.

64. Y. Zhang, W.-X. Feng, Y.-M. Legrand, C. T. Supuran, C.-Y. Su, M. Barboiu, Chem. Commun., 2016, 52, 13768-13770.

TOC image and text

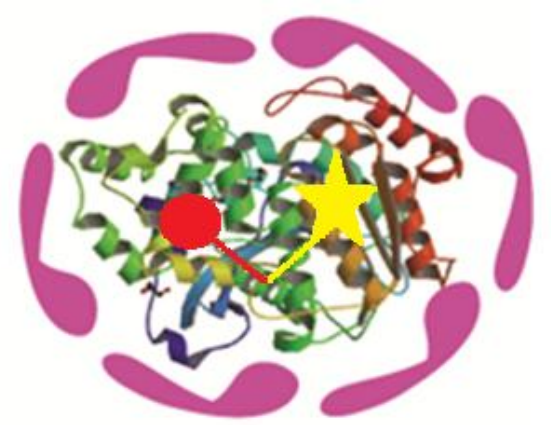

Dynamic constitutional strategies for enzyme activity inhibition and activation are described by using active dynamic molecular libraries and dynamic molecular frameworks 